Article

\title{
Thermodynamic Relations for the Entropy and Temperature of Multi-Horizon Black Holes
}

\author{
Wei Xu ${ }^{1,2, *}$, Jia Wang ${ }^{2}$ and Xin-he Meng ${ }^{2,3}$ \\ ${ }^{1}$ School of Physics, Huazhong University of Science and Technology, Wuhan 430074, China \\ ${ }^{2}$ School of Physics, Nankai University, Tianjin 300071, China; \\ E-Mails: wangjia2010@mail.nankai.edu.cn (J.W.); xhm@nankai.edu.cn (X.M.) \\ ${ }^{3}$ State Key Laboratory of ITP, ITP-CAS, Beijing 100190, China \\ * Author to whom correspondence should be addressed; E-Mail: xuweifuture@ gmail.com; \\ Academic Editor: Lorenzo Iorio
}

Received: 12 October 2014 / Accepted: 28 January 2015 / Published: 2 February 2015

\begin{abstract}
We present some entropy and temperature relations of multi-horizons, even including the "virtual" horizon. These relations are related to the product, division and sum of the entropy and temperature of multi-horizons. We obtain the additional thermodynamic relations of both static and rotating black holes in three- and four-dimensional (A)dS spacetime. Especially, a new dimensionless, charge-independence and $T_{+} S_{+}=T_{-} S_{-}$-like relation is presented. This relation does not depend on the mass, electric charge, angular momentum and cosmological constant, as it is always a constant. These relations lead us to obtaining some interesting thermodynamic bounds of entropy and temperature, including the Penrose inequality, which is the first geometrical inequality of black holes. Besides, based on these new relations, one can obtain the first law of thermodynamics and the Smarr relation for all horizons of a black hole.
\end{abstract}

Keywords: black holes; thermodynamic relations; thermodynamic bound; thermodynamic laws; classical theories of gravity

PACS classifications: 04.70.Bw, 04.70.Dy, 04.70.-s 


\section{Introduction}

One of the central issues in quantum gravity is to understand the entropy of a black hole microscopically. Significant insights have been achieved for four- and five- dimensional, supersymmetric, asymptotically flat, multi-charged black holes [1], where the microscopic degrees of freedom can be explained in terms of a two-dimensional conformal field theory. Another important work has focused on the microscopic entropy of extreme rotating solutions [2] via the Kerr/CFT correspondence. Besides, it is found in [3-6] that black hole entropy and the number of micro-states result in being a function of the principal quantum number of the quasi-normal modes (QNMs), which can represent the "quantum level" of a black hole. This seems consistent with various quantum gravity models, where the spacetime is fundamentally discrete and leads to another possible way for studying black hole entropy microscopically. However, the detailed microscopic origin of the entropy of non-extremal, rotating, charged black holes remains an open problem now. For this issue, much attention has been paid to the additional entropy relations of black holes recently [7-29].

The entropy products of multi-horizons [7-25] are firstly introduced, which are expected to be not only expressed solely in terms of the quantized charges, including the electric charge $Q$, the angular momentum $J$ and the cosmological constant $\Lambda$ (which can be treated as pressure after explaining the mass of the black hole as enthalpy rather than the internal energy of the system), but to also have mass independence. This study is generalizable to many theories, including the super-gravity model [7-11], Einstein gravity [12-20,25] and other modified gravity models [9,21-25] in both four and higher dimensions. It is always independent of the mass of the black hole, as shown in [7-11,13-16,18,23,24,27,28]. However, the mass independence of the entropy product fails in some asymptotically non-flat spacetime or modified gravity theories [12,17,21,22,25]. Hence, the "part" entropy product $[17,25,26]$ i.e., $\sum_{1 \leq i<j \leq D}\left(S_{i} S_{j}\right)^{\frac{1}{d-2}}$ (here and below, $D$ and $d$ are denoted as the number of horizons and the dimensions, respectively, in this paper) and the entropy sum [25,27-29] are introduced, which always are independent of the mass of the black hole in (A)dSspacetime. These two never depend on the electric charge $Q$ and angular momentum $J$, but only depend on the cosmological constant and the constants characterizing the strength of these extra matter fields. Beside, it is found that the entropy product and the "part" entropy product belong to the same kind of entropy relation, as their mass independence of entropy hold complementarily in the theory without the Maxwell field [25]. Furthermore, the "part" entropy product and entropy sum of the Schwarzschild-de-Sitter black hole are actually equal [25], when only the effect of the physical horizons are considered, as they both can be simplified into mass-independent entropy relations of a physical horizon [17,25].

Actually, there exist several reasons why people study the entropy relations of multi-horizons and the physics near other horizons. Firstly, it is shown in [30-32] that the Green functions are sensitive to the geometry near all of the black hole horizons and not just the outermost one. Hence, the entropy at each horizon can therefore be expected to play a role in governing the properties of the black hole at the microscopic level. Secondly, the entropy inequalities of multi-horizons of four-dimensional general axisymmetric stationary solutions in the Einstein-Maxwell theory [33-39] are interpreted as a general criterion for extremality [40], which also results in a no-go theorem for the possibility of force balance between two rotating black holes [41]. This makes the physics at each horizon more intriguing. Besides, 
the effect of the other horizons is necessary in order to preserve the mass independence [17,27-29]. Only including this effect can these additional equalities of multi-horizons of black holes be "universal".

In this paper, we study the additional thermodynamic relations of black holes with multi-horizons, in order further study the understanding of the origin of black hole entropy at the microscopic level. The thermodynamic relations are related to the thermodynamic quantities, especially for the temperature and entropy for two physical horizons [11,12,15,21,42-48]. Other quantities, like the charge potential and angular velocity, are sometime considered, as well [11,19-21,48]. These thermodynamic relations were understood well and physically, which were also linked closely with the entropy product via their holographic description, i.e., the thermodynamic method of the black hole/CFT correspondence [15,42-47]. The black hole/CFT (BH/CFT) correspondence focuses on black holes with two physical horizons, and its key point is that the inner horizon thermodynamics may play an essential role in setting up the BH/CFT correspondence. From the thermodynamics laws of both horizons (one need note that it is a so-called "thermodynamics law"-like relation for the inner horizon), it is straightforward to read the thermodynamics of the left- and right-mover and the corresponding dual temperatures. This method has only been used for the black holes in the Einstein (and Maxwell) gravity with two physical horizons up till now [15,42-47]. However, only for the two physical horizons' case, one can prove that the first law of thermodynamics of the outer horizon always indicates that of the inner horizon under reasonable assumption. Moreover, only for this class of gravity theory, which is diffeomorphism invariant, is its dual two-dimensional CFT required to have $c_{R}=c_{L}$. As a result, the central charge being the same is equivalent to the condition $T_{+} S_{+}=T_{-} S_{-}$in the thermodynamics method or, equivalently, the condition that the entropy product $S_{+} S_{-}$is mass-independent $[12,15,42-47]$, where $T_{ \pm}, S_{ \pm}$are the outer and inner horizon temperatures and entropies, respectively. Therefore, the thermodynamics relations $T_{+} S_{+}=T_{-} S_{-}$(equivalently, entropy product $S_{+} S_{-}$being mass independent) may be taken as the criterion, whether there is a two-dimensional CFT dual for the black holes in the Einstein gravity and other diffeomorphism invariant gravity theories [15,42-47]. Namely, these thermodynamics relations really give a clue to the microscopics of black holes. When the discussion generalizes to some cases of black holes with more than two horizons, the thermodynamics relations $T_{+} S_{+}=T_{-} S_{-}$(equivalently, entropy product $S_{+} S_{-}$being mass independent) break down [12,15,17,21,25], and there are even only two physical horizons. Hence, we aim to study the additional thermodynamic relations of black holes with more than two horizons (other than the case of two physical horizons), in order to improve the understanding of the origin of black hole entropy at the microscopic level for these (A)dS black holes.

Mainly, we present some general entropy and temperature relations of multi-horizons, even the "virtual" horizon. Actually, studying these kinds of relations for understanding the internal physics of black holes is not a new idea. A discussion of a Kerr black hole had been presented in [48] in 1992. These relations are related to the product, division and sum of the entropy and temperature of multi-horizons. We generalize them to the similar relations of static and rotating black holes in three and four dimension. Some general thermodynamic relations are constructed and found to hold for both AdSand dS black holes. Moreover, a new dimensionless and charge-independence relation $\left(\sum_{i=1}^{D} T_{i} S_{i}\right) \times\left(\sum_{i=1}^{D} \frac{1}{T_{i} S_{i}}\right)$ is presented, which is the generalizing of the $T_{+} S_{+}=T_{-} S_{-}$relations for two horizons. This does not depend on the mass, electric charge, angular momentum and cosmological 
constant, as it is always a constant for black holes, as shown in the present paper. This relation is expected to be helpful of constructing the BH/CFT correspondence for more than two horizons, in order to understand the black hole entropy microscopically. Besides, based on these relations, we obtain some interesting thermodynamic bounds of entropy and temperature, including the Penrose inequality, which is the first geometrical inequality of black holes (event horizon) (see [49] for a review). We also get the Penrose-like inequalities for other horizons. Moreover, these thermodynamic relations can lead to the thermodynamic law and Smarr relation of the event horizon and the Cauchy horizon of black holes. We take the case of a Kerr black hole as a detailed example. This discussion is generalized to a Kerr-Newman black hole, as well.

This paper is organized as follows. In the next section, we will investigate the thermodynamic relations of static black holes in four-dimensional (A)dS spacetime. In Section 3, the thermodynamic relations of rotating black holes in three dimensions and four dimensions are presented. Using these thermodynamic relations, we obtain some interesting thermodynamic bounds in Section 4 and the first law and Smarr relation of black holes in Section 5, respectively. Section 6 is devoted to the conclusions and discussions.

\section{Thermodynamic Relations of Static (A)dS Black Holes}

In this section, we investigate many thermodynamic relations of static black holes in four-dimensional (A)dS spacetime. Among these relations, a new dimensionless and charge-independence relation $\left(\sum_{i=1}^{D} T_{i} S_{i}\right) \times\left(\sum_{i=1}^{D} \frac{1}{T_{i} S_{i}}\right)$ is presented, which is the generalizing of the $T_{+} S_{+}=T_{-} S_{-}$relations.

\subsection{Thermodynamic Relations of Schwarzschild-(A)dS Black Holes}

We firstly give the thermodynamic relations of Schwarzschild-(A)dS black holes in detail. We begin with the line element:

$$
d s^{2}=-f(r) d t^{2}+\frac{d r^{2}}{f(r)}+r^{2}\left(d \theta^{2}+\sin ^{2} d \varphi^{2}\right) .
$$

For four-dimensional a Schwarzschild-de-Sitter black hole, the horizon function is:

$$
f(r)=1-\frac{2 M}{r}-\frac{\Lambda r^{2}}{3}
$$

with $M$ being the mass of the black hole and $\Lambda=\frac{1}{L^{2}}$ being the cosmological constant. The three black hole horizons are [17]:

$$
\begin{aligned}
& r_{E}=2 L \sin \left(\frac{1}{3} \arcsin \left(\frac{3 M}{L}\right)\right) \\
& r_{C}=2 L \sin \left(\frac{1}{3} \arcsin \left(\frac{3 M}{L}\right)+\frac{2 \pi}{3}\right) \\
& r_{V}=2 L \sin \left(\frac{1}{3} \arcsin \left(\frac{3 M}{L}\right)-\frac{2 \pi}{3}\right),
\end{aligned}
$$


where $r_{E}, r_{C}$ and $r_{V}$ represent the event horizon, cosmological horizon and "virtual" horizon, respectively. The Bekenstein area entropy [50] and Hawking temperature [51] of each horizon are $S_{i}=A_{i} / 4=\pi r_{i}^{2}$ and:

$$
T_{i}=\frac{f^{\prime}\left(r_{i}\right)}{4 \pi}=\frac{L^{2}-r_{i}^{2}}{4 \pi L^{2} r_{i}},(i=E, C, V)
$$

respectively, where $f^{\prime}(r)$ denotes the derivative function of $f(r)$ with respect to $r$.

We firstly revisit some known entropy relations: the mass-dependence entropy product [17],

$$
S_{E} S_{C} S_{V}=36 \pi^{3} M^{2} L^{4}
$$

the equal and mass independence "part” entropy product and entropy sum [17,25,27],

$$
\begin{aligned}
& S_{E} S_{C}+S_{E} S_{V}+S_{C} S_{V}=9 \pi^{2} L^{4} \\
& S_{E}+S_{C}+S_{V}=6 \pi L^{2},
\end{aligned}
$$

which can lead into the entropy relations of two physical horizons having mass independence [17,25]:

$$
S_{E}+S_{C}+\sqrt{S_{E} S_{C}}=3 \pi L^{2} .
$$

Based on these entropy relations, one can construct and calculate more relations. For example, the mass-independence case:

$$
\begin{aligned}
S_{E}^{2}+S_{C}^{2}+S_{V}^{2} & =\left(S_{E}+S_{C}+S_{V}\right)^{2}-2\left(S_{E} S_{C}+S_{E} S_{V}+S_{C} S_{V}\right) \\
& =18 \pi^{2} L^{4}
\end{aligned}
$$

and some mass-dependence cases:

$$
\begin{aligned}
& \frac{1}{S_{E}}+\frac{1}{S_{C}}+\frac{1}{S_{V}}=\frac{1}{4 \pi M^{2}}, \\
& \frac{1}{S_{E} S_{C}}+\frac{1}{S_{E} S_{V}}+\frac{1}{S_{C} S_{V}}=\frac{1}{6 \pi^{2} M^{2} L^{2}}, \\
& \frac{S_{C} S_{V}}{S_{E}}+\frac{S_{V} S_{E}}{S_{C}}+\frac{S_{E} S_{C}}{S_{V}}=\frac{9 \pi L^{4}}{4 M^{2}}-12 \pi L^{2}, \\
& \frac{S_{C}+S_{V}}{S_{E}}+\frac{S_{V}+S_{E}}{S_{C}}+\frac{S_{E}+S_{C}}{S_{V}}=\frac{3 L^{2}}{2 M^{2}}-3,
\end{aligned}
$$

following the same procedure.

Then, we turn to the temperature relations and study the product, division and sum of the temperature of multi-horizons. In order to construct similar relations, we need to introduce the relationship of three horizons:

$$
\begin{aligned}
& r_{E}+r_{C}+r_{V}=0, \quad r_{E}+r_{C}+r_{V}=-6 M L^{2} \\
& r_{E} r_{C}+r_{E} r_{V}+r_{C} r_{V}=-3 L^{2}
\end{aligned}
$$


and by using this, one can find the mass-independence case:

$$
\begin{aligned}
& T_{E} T_{C}+T_{C} T_{V}+T_{V} T_{E}=0, \\
& \frac{1}{T_{E}}+\frac{1}{T_{C}}+\frac{1}{T_{V}}=0, \\
& \frac{T_{C}+T_{V}}{T_{E}}+\frac{T_{V}+T_{E}}{T_{C}}+\frac{T_{E}+T_{C}}{T_{V}}=-3,
\end{aligned}
$$

and some mass-dependence cases:

$$
\begin{aligned}
& T_{E}+T_{C}+T_{V}=\frac{1}{8 \pi M}, \\
& T_{E}^{2}+T_{C}^{2}+T_{V}^{2}=\frac{1}{64 \pi^{2} M^{2}}, \\
& \frac{T_{C} T_{V}}{T_{E}}+\frac{T_{V} T_{E}}{T_{C}}+\frac{T_{E} T_{C}}{T_{V}}=-\frac{1}{4 \pi M}, \\
& \frac{1}{T_{E} T_{C}}+\frac{1}{T_{C} T_{V}}+\frac{1}{T_{V} T_{E}}=\frac{12 \pi^{2} L^{4}}{9 M^{2}-L^{2}}, \\
& T_{E} T_{C} T_{V}=\frac{6 M}{L^{4}}-\frac{2}{3 M L^{2}} .
\end{aligned}
$$

One can conclude that:

$$
\frac{1}{S_{E}}+\frac{1}{S_{C}}+\frac{1}{S_{V}}=16 \pi\left(T_{E}+T_{C}+T_{V}\right)^{2}=16 \pi\left(T_{E}^{2}+T_{C}^{2}+T_{V}^{2}\right)
$$

For the $T_{+} S_{+}=T_{-} S_{-}$-like relations, one can find:

$$
\begin{aligned}
& T_{E} S_{E}+T_{C} S_{C}+T_{V} S_{V}=\frac{9 M}{2}, \\
& \frac{1}{T_{E} S_{E}}+\frac{1}{T_{C} S_{C}}+\frac{1}{T_{V} S_{V}}=\frac{2}{M},
\end{aligned}
$$

which are both dependent of mass and different from the results for flat black holes with two physical horizons.

For the discussion of a Schwarzschild-AdS black hole, one can find that the above relations are all universal after taking the transitions $L \rightarrow i L$. Namely, instead $L$ of $\frac{1}{\Lambda}$, all of the above relations always hold for a Schwarzschild-(A)dS black hole. Moreover, we obtain the dimensionless relation:

$$
\left(T_{E} S_{E}+T_{C} S_{C}+T_{V} S_{V}\right)\left(\frac{1}{T_{E} S_{E}}+\frac{1}{T_{C} S_{C}}+\frac{1}{T_{V} S_{V}}\right)=9 .
$$

One will find that this one is always charge independent, as shown again in the cases for ReissnerNordström-(A)dS black holes.

\subsection{Thermodynamic Relations of Reissner-Nordström-(A)dS Black Holes}

For further studying, we list the thermodynamic relations of Reissner-Nordström-(A)dS black holes following the same procedure. The horizon function is:

$$
f(r)=1-\frac{2 M}{r}-\frac{\Lambda r^{2}}{3}+\frac{Q^{2}}{r^{2}}
$$


The four black hole horizons are $r_{1}, r_{2}, r_{3}$ and $r_{4}$. The Bekenstein area-entropy [50] and Hawking temperature [51] of each horizon are $S_{i}=A_{i} / 4=\pi r_{i}^{2}$ and:

$$
T_{i}=\frac{f^{\prime}\left(r_{i}\right)}{4 \pi}=\frac{1}{4 \pi}\left(\frac{1}{r_{i}}-\Lambda r_{i}-\frac{Q^{2}}{r_{i}^{3}}\right),(i=1,2,3,4)
$$

respectively.

We still firstly revisit some known entropy relations: the mass-independence entropy product and entropy sum $[17,27]$ :

$$
\begin{aligned}
& S_{1} S_{2} S_{3} S_{4}=\frac{9 \pi^{4} Q^{4}}{\Lambda^{2}} \\
& S_{1}+S_{2}+S_{3}+S_{4}=\frac{6 \pi}{\Lambda} .
\end{aligned}
$$

Based on these entropy relations and the following relationship of four horizons:

$$
\begin{aligned}
& r_{1}+r_{2}+r_{3}+r_{4}=0, \quad r_{1} r_{2}+r_{1} r_{3}+r_{1} r_{4}+r_{2} r_{3}+r_{2} r_{4}+r_{3} r_{4}=-\frac{3}{\Lambda}, \\
& r_{1} r_{2} r_{3} r_{4}=-\frac{3 Q^{2}}{\Lambda}, \quad r_{1} r_{2} r_{3}+r_{1} r_{3} r_{4}+r_{1} r_{2} r_{4}+r_{2} r_{3} r_{4}=-\frac{6 M}{\Lambda},
\end{aligned}
$$

one can also construct and calculate more relations. For example, the mass-independence case:

$$
\begin{aligned}
& S_{1} S_{2}+S_{1} S_{3}+S_{2} S_{3}+S_{1} S_{4}+S_{2} S_{4}+S_{3} S_{4}=\frac{9 \pi^{2}}{\Lambda^{2}}-\frac{6 \pi^{2} Q^{2}}{\Lambda}, \\
& \frac{1}{S_{1} S_{2}}+\frac{1}{S_{1} S_{3}}+\frac{1}{S_{2} S_{3}}+\frac{1}{S_{1} S_{4}}+\frac{1}{S_{3} S_{4}}+\frac{1}{S_{2} S_{4}}=\frac{1}{\pi^{2} Q^{4}}-\frac{2 \Lambda}{3 \pi^{2} Q^{2}}
\end{aligned}
$$

and some mass-dependence cases:

$$
\begin{aligned}
& S_{1} S_{2} S_{3}+S_{1} S_{3} S_{4}+S_{2} S_{3} S_{4}+S_{1} S_{2} S_{4}=-\frac{6 \pi^{3}\left(M^{2}+3 Q^{2}\right)}{\Lambda^{2}}, \\
& S_{1}^{2}+S_{2}^{2}+S_{3}^{2}+S_{4}^{2}=\frac{18 \pi^{2}}{\Lambda^{2}}+\frac{12 \pi^{2} Q^{2}}{\Lambda}, \\
& \frac{1}{S_{1}}+\frac{1}{S_{2}}+\frac{1}{S_{3}}+\frac{1}{S_{4}}=-\frac{2\left(M^{2}+3 Q^{2}\right)}{3 \pi Q^{4}}, \\
& \frac{S_{2} S_{3} S_{4}}{S_{1}}+\frac{S_{1} S_{3} S_{4}}{S_{2}}+\frac{S_{1} S_{2} S_{4}}{S_{3}}+\frac{S_{2} S_{3} S_{4}}{S_{4}}=\frac{4 \pi^{2} M^{4}}{\Lambda^{2} Q^{4}}+\frac{24 \pi^{2} M^{2}}{\Lambda^{2} Q^{2}}+\frac{12 \pi^{2} Q^{2}}{\Lambda}+\frac{18 \pi^{2}}{\Lambda^{2}}, \\
& \frac{S_{2}+S_{3}+S_{4}}{S_{1}}+\frac{S_{1}+S_{3}+S_{4}}{S_{2}}+\frac{S_{1}+S_{2}+S_{4}}{S_{3}}+\frac{S_{1}+S_{2}+S_{3}}{S_{4}}=-\frac{4 M^{2}}{\Lambda Q^{4}}-\frac{12}{\Lambda Q^{2}}-4,
\end{aligned}
$$

following the same procedure.

Then, we turn to the temperature relations and study the product, division and sum of the temperature of multi-horizons. However, one can only find some mass-dependence cases:

$$
\begin{aligned}
& T_{1}+T_{2}+T_{3}+T_{4}=\frac{2 M}{\pi Q^{2}}-\frac{13 M^{3}}{3 \pi Q^{4}} \\
& T_{1} T_{2} T_{3}+T_{1} T_{3} T_{4}+T_{2} T_{3} T_{4}+T_{1} T_{2} T_{4}=\frac{7 \Lambda M^{3}}{36 \pi^{3} Q^{4}} .
\end{aligned}
$$

These relations are not universal, as one anticipates. 
For the $T_{+} S_{+}=T_{-} S_{-}$-like relations, one can find:

$$
\begin{aligned}
& T_{1} S_{1}+T_{2} S_{2}+T_{3} S_{3}+T_{4} T_{4}=16 M \\
& \frac{1}{T_{1} S_{1}}+\frac{1}{T_{2} S_{2}}+\frac{1}{T_{3} S_{3}}+\frac{1}{T_{4} S_{4}}=0
\end{aligned}
$$

which are both from the results for asymptotically flat black holes with two physical horizons and that for Schwarzschild-(A)dS black holes.

One can note that all of the above relations always hold for both AdS and dS black holes. Moreover, we again obtain the dimensionless and charge-independence relation:

$$
\left(T_{1} S_{1}+T_{2} S_{2}+T_{3} S_{3}+T_{4} S_{4}\right)\left(\frac{1}{T_{1} S_{1}}+\frac{1}{T_{2} S_{2}}+\frac{1}{T_{3} S_{3}}+\frac{1}{T_{4} S_{4}}\right)=0
$$

for Reissner-Nordström-(A)dS black holes. This also belongs to the kind of thermodynamic relation $\left(\sum_{i=1}^{D} T_{i} S_{i}\right) \times\left(\sum_{i=1}^{D} \frac{1}{T_{i} S_{i}}\right)$, with $D=4$ being the number of horizons, which is always a constant. Consider the degenerated case $\Lambda=0$, i.e., a Reissner-Nordström black hole; the relation (16) reduces to:

$$
\left(T_{1} S_{1}+T_{2} S_{2}\right)\left(\frac{1}{T_{1} S_{1}}+\frac{1}{T_{2} S_{2}}\right)=0,
$$

$D=2$ in this case, one is an event horizon and another is a Cauchy horizon, which is also a constant. This is the generalizing of the $T_{+} S_{+}=T_{-} S_{-}$relations; hence, it is expected to be helpful for constructing the $\mathrm{BH} / \mathrm{CFT}$ correspondence for more than two horizons.

\section{Thermodynamic Relation of Rotating Black Holes}

In this section, we focus on the thermodynamic relation of rotating black holes in three and four dimensions, especially for the the dimensionless relation. Namely, we will mainly recheck the generalizing of the $T_{+} S_{+}=T_{-} S_{-}$relations for more than two horizons, i.e., $\left(\sum_{i=1}^{D} T_{i} S_{i}\right) \times\left(\sum_{i=1}^{D} \frac{1}{T_{i} S_{i}}\right)$ for rotating black holes. It is still a constant, which is charge independent (mass independent, charge independent and angular momentum independent).

\subsection{Thermodynamic Relation of Kerr (and Newman) Black Holes}

The Kerr metric in Boyer-Lindquist coordinates is:

$$
\begin{aligned}
d s^{2}= & -\frac{\Delta-a^{2} \sin ^{2} \theta}{\Sigma} d t^{2}-2 a \sin ^{2} \theta\left(\frac{r^{2}+a^{2}-\Delta}{\Sigma}\right) d t d \phi \\
& +\left(\frac{\left(r^{2}+a^{2}\right)^{2}-\Delta a^{2} \sin ^{2} \theta}{\Sigma}\right) \sin ^{2} \theta d^{2} \phi+\frac{\Sigma}{\Delta} d r^{2}+\Sigma d^{2} \theta
\end{aligned}
$$

where:

$$
\begin{gathered}
\Sigma=r^{2}+a^{2} \cos ^{2} \theta, \\
\Delta(r)=r^{2}-2 M r+a^{2} .
\end{gathered}
$$


The zeros of $\Delta(r)$ correspond to the event horizon and Cauchy horizon, denoted as $r_{+}$and $r_{-}$:

$$
\begin{aligned}
& r_{+}=M+\sqrt{M^{2}-a^{2}}, \\
& r_{-}=M-\sqrt{M^{2}-a^{2}} .
\end{aligned}
$$

Since the metric is not diagonal, the Hawking temperature [51] should be $T=\frac{\kappa}{2 \pi}$. The surface gravity $\kappa$ and temperature $T$ are:

$$
\begin{aligned}
\kappa_{ \pm} & =\frac{r_{ \pm}-r_{\mp}}{2\left(r_{ \pm}^{2}+a^{2}\right)}, \\
T_{ \pm} & =\frac{r_{ \pm}-r_{\mp}}{4 \pi\left(r_{ \pm}^{2}+a^{2}\right)} .
\end{aligned}
$$

Additionally, the Bekenstein area entropy [50] for each horizon is:

$$
S_{ \pm}=\frac{A_{ \pm}}{4}=\pi\left(r_{ \pm}^{2}+a^{2}\right)
$$

with:

$$
T_{ \pm} S_{ \pm}=\frac{r_{ \pm}-r_{\mp}}{4}
$$

Obviously, these lead to the entropy product:

$$
S_{+} S_{-}=4 \pi^{2} J^{2}
$$

entropy sum:

$$
S_{+}+S_{-}=4 \pi M^{2}
$$

entropy minus:

$$
S_{+}-S_{-}=2 \pi M\left(r_{+}-r_{-}\right)= \pm 8 \pi M T_{ \pm} S_{ \pm},
$$

and the mass-dependent relation:

$$
\frac{1}{S_{+}}+\frac{1}{S_{-}}=\frac{S_{+}+S_{-}}{S_{+} S_{-}}=\frac{M^{2}}{\pi J^{2}},
$$

where $J=a M$ is the angular momentum. The angular velocity is:

$$
\Omega_{ \pm}=\frac{a}{r_{ \pm}^{2}+a^{2}}=\frac{\pi J}{M S_{ \pm}}
$$

based on which, we can obtain:

$$
\Omega_{+}+\Omega_{-}=\frac{M}{J}
$$

On the other hand, we find:

$$
\begin{aligned}
& T_{+} S_{+}+T_{-} S_{-}=0 \\
& \frac{1}{T_{+} S_{+}}+\frac{1}{T_{-} S_{-}}=0
\end{aligned}
$$


thus:

$$
\left(T_{+} S_{+}+T_{-} S_{-}\right)\left(\frac{1}{T_{+} S_{+}}+\frac{1}{T_{-} S_{-}}\right)=0 .
$$

which shows that the charge-independence of dimensionless relation $\left(\sum_{i=1}^{D} T_{i} S_{i}\right) \times\left(\sum_{i=1}^{D} \frac{1}{T_{i} S_{i}}\right)$ holds.

For a Kerr-Newman black hole, one can substitute $a^{2}$ with $a^{2}+Q^{2}$, where $Q$ is the electric charge; apparently the relation (29) still holds.

\subsection{Thermodynamic Relation of a BTZ Black Hole}

Considering a BTZ black hole [52], the metric reads:

$$
d s^{2}=-f(r) d t^{2}+\frac{d r^{2}}{f(r)}+r^{2}\left(N^{\phi}(r) d t+d \phi\right)^{2}
$$

where the cosmological constant $\Lambda=-\frac{1}{l^{2}}$, and the horizon function $f(r)$ and the angular velocity $N^{\phi}(r)$ :

$$
\begin{aligned}
f(r) & =-M+\frac{r^{2}}{\ell^{2}}+\frac{J^{2}}{4 r^{2}}, \\
N^{\phi}(r) & =-\frac{J}{2 r^{2}}
\end{aligned}
$$

where $M$ and $J$ are the mass and angular momentum of the black hole, respectively. The horizon function $f(r)$ has four zeros:

$$
\begin{aligned}
& r_{1}=\sqrt{\left(1+\sqrt{1-\left(\frac{J}{M \ell}\right)^{2}}\right) M \ell}, \quad r_{2}=-\sqrt{\left(1+\sqrt{1-\left(\frac{J}{M \ell}\right)^{2}}\right) M \ell} \\
& r_{3}=\sqrt{\left(1-\sqrt{1-\left(\frac{J}{M \ell}\right)^{2}}\right) M \ell,} \quad r_{4}=-\sqrt{\left(1-\sqrt{1-\left(\frac{J}{M \ell}\right)^{2}}\right) M \ell}
\end{aligned}
$$

where $r_{1}$ and $r_{3}$ correspond to the event horizon and Cauchy horizon, i.e., physical horizons, while $r_{2}$ and $r_{4}$ represent the negative and un-physical "virtual" horizons, which often are discarded in the literature.

As discussed in [52], the Bekenstein "area" entropy [50] is equal to twice the perimeter length of the horizon,

$$
S_{i}=4 \pi r_{i}
$$

and the Hawking temperature [51] is:

$$
T_{i}=\left(\frac{\partial S_{i}}{\partial M}\right)^{-1}
$$

Firstly, we discuss the physical horizons only, i.e., $r_{1}$ and $r_{3}$. With the help of a computer algebra system (CAS), one can get:

$$
T_{1} S_{1}+T_{3} S_{3}=0
$$


meanwhile, $T_{1} S_{1}$ or $T_{3} S_{3}$ does not equal zero explicitly, so obviously:

$$
\left(T_{1} S_{1}+T_{3} S_{3}\right)\left(\frac{1}{T_{1} S_{1}}+\frac{1}{T_{3} S_{3}}\right)=0 .
$$

Secondly, we should include the un-physical, "virtual" horizons $r_{2}$ and $r_{4}$. We note that $r_{1}$ and $r_{2}$ have the opposite sign, as well as $r_{3}$ and $r_{4}$. Therefore, $S_{2}, S_{4}, T_{2}$ and $T_{4}$ have a minus sign to their counterpart, respectively, as we discussed in the last paragraph. However, $T_{2} S_{2}+T_{4} S_{4}=T_{1} S_{1}+T_{3} S_{3}=0$, and apparently:

$$
\left(T_{1} S_{1}+T_{2} S_{2}+T_{3} S_{3}+T_{4} S_{4}\right)\left(\frac{1}{T_{1} S_{1}}+\frac{1}{T_{2} S_{2}}+\frac{1}{T_{3} S_{3}}+\frac{1}{T_{4} S_{4}}\right)=0 .
$$

Again, the dimensionless relation $\left(\sum_{i=1}^{D} T_{i} S_{i}\right) \times\left(\sum_{i=1}^{D} \frac{1}{T_{i} S_{i}}\right)$ is charge independent.

\section{Thermodynamic Relations and Thermodynamic Bound}

In this section, based on the thermodynamic relations presented in this paper, we obtain some thermodynamic bounds of the thermodynamic quantities, including the entropy and temperature. From the entropy bound, one can get the area bound. Especially for the upper area bound of the event horizon of black holes, one can find that it is actually the exact Penrose inequality of a black hole, which is the first geometrical inequality of a black hole (see [49] for a review).

\subsection{Thermodynamic Bound for a Schwarzschild-dS Black Hole}

Firstly, we show the thermodynamic bound for a Schwarzschild-dS black hole. Consider the thermodynamic relations of a Schwarzschild-dS black hole shown in Section 2.1. We will only focus on the cases with:

$$
\frac{3 M}{L} \leq 1
$$

which leads to three real horizons according to Equation (1). Then, we will find that the entropy and temperature for three horizons are all real (for $\frac{3 M}{L}>1$, one will only find one real horizon). Especially for the event horizon, cosmological horizon and the "virtual" horizon, we know that $0 \leq r_{E} \leq L \leq$ $r_{C} \leq\left|r_{V}\right| \leq 2 L$ and $r_{V}<0$; hence, $0 \leq S_{E} \leq S_{C} \leq S_{V} \leq 4 \pi L^{2}$ and $T_{E} \geq 0, T_{C} \leq 0, T_{V} \geq 0$ (note that the Hawking temperature of the cosmological horizon is $\hat{T}_{C}=-T_{C} \geq 0$ ).

From thermodynamic relation Equation (5), we get:

$$
0 \leq 3 S_{E} \leq\left(S_{E}+S_{C}+\sqrt{S_{E} S_{C}}\right)=3 \pi L^{2} \leq 3 S_{C},
$$

and:

$$
0 \leq S_{C} \leq 3 \pi L^{2}
$$

which together give:

$$
0 \leq S_{E} \leq \pi L^{2} \leq S_{C} \leq 3 \pi L^{2}
$$


As $0 \leq\left(S_{C}+S_{E}\right) \leq 3 \pi L^{2}$, from the entropy sum Equation (4), we find:

$$
S_{V} \geq 3 \pi L^{2}
$$

In total, we obtain the entropy bound of the event horizon, the cosmological horizon and the negative horizon:

$$
S_{E} \in\left[0, \pi L^{2}\right], \quad S_{C} \in\left[\pi L^{2}, 3 \pi L^{2}\right], \quad S_{V} \in\left[3 \pi L^{2}, 4 \pi L^{2}\right] .
$$

Additionally, the area entropy leads to the area bounds:

$$
\sqrt{\frac{A_{E}}{16 \pi}} \in\left[0, \frac{L}{2}\right], \quad \sqrt{\frac{A_{C}}{16 \pi}} \in\left[\frac{L}{2}, \sqrt{\frac{3}{4}} L\right], \quad \sqrt{\frac{A_{V}}{16 \pi}} \in\left[\sqrt{\frac{3}{4}} L, L\right],
$$

which are all geometrical bounds of black hole horizons, as parameter $L$ is actually the cosmological radius.

On the other hand, consider the mass-dependence thermodynamic relations of entropy Equation (7); we can also find:

$$
S_{E} \geq 4 \pi M^{2}
$$

which leads to the Penrose-like inequality:

$$
\sqrt{\frac{A_{E}}{16 \pi}} \geq \frac{M}{4}
$$

Inserting condition Equation (33) into the area bound, we can obtain other Penrose-like inequalities:

$$
\sqrt{\frac{A_{C}}{16 \pi}} \geq \frac{3 M}{2}, \quad \sqrt{\frac{A_{V}}{16 \pi}} \geq 3 \sqrt{\frac{3}{4}} M .
$$

Furthermore, we can get the temperature bound from Equation (8), i.e.,

$$
T_{i} \leq \frac{1}{8 \pi M}(i=E, V) ; \quad\left|T_{C}\right| \leq \frac{1}{8 \pi M}
$$

\subsection{Thermodynamic Bound for Kerr Black Holes}

For black holes in rotating spacetime, we take the thermodynamic relations of the Kerr black holes as an example, which is shown in Section 3.1. From Equation (18), the existence of black hole horizons leads to the famous Kerr bound:

$$
\frac{M}{a} \geq 1
$$

or equivalently, $M^{2} \geq J$. Besides, $r_{+} \geq r_{-}$leads to $S_{+} \geq S_{-} \geq 0$. Then, the entropy product Equation (22) results in:

$$
S_{+} \geq \sqrt{S_{+} S_{-}}=2 \pi J, \quad S_{-} \leq \sqrt{S_{+} S_{-}}=2 \pi J .
$$


Additionally, from the entropy sum Equation (23), we obtain:

$$
2 \pi M^{2}=\frac{\left(S_{+}+S_{-}\right)}{2} \leq S_{+} \leq\left(S_{+}+S_{-}\right)=4 \pi M^{2}, \quad S_{-} \leq \frac{\left(S_{+}+S_{-}\right)}{2}=2 \pi M^{2} .
$$

These together give the entropy bound of the event horizon and the Cauchy horizon:

$$
S_{+} \in\left[2 \pi M^{2}, 4 \pi M^{2}\right], \quad S_{-} \in[0,2 \pi J] .
$$

Note that the Kerr bound relation is used here. Because of the area entropy Equation (20), we also get the area bound of the event horizon and the Cauchy horizon:

$$
\sqrt{\frac{A_{+}}{16 \pi}} \in\left[\frac{M}{\sqrt{2}}, M\right], \quad \sqrt{\frac{A_{-}}{16 \pi}} \in\left[0, \sqrt{\frac{J}{2}}\right] .
$$

Note that the upper bound of the event horizon is actually the exact Penrose inequality of the black hole.

Actually, for black holes with real horizons (e.g., Kerr-Newman black holes, Reissner-Nordström-dS black holes, etc.), we can follow a similar procedure to obtain the bound of thermodynamic quantities. However, if the black hole horizon is not real, the method presented here fails.

\section{Thermodynamic Relations and Thermodynamic Laws}

The thermodynamic bounds in the preceding section can be seen as the application of the thermodynamic relations, while how they link to the thermodynamics of black holes seems to be still unclear. However, based on these thermodynamic relations, as well, one can obtain the thermodynamic law and Smarr relation of all horizons of black holes. In this section, we only take the discussion about the event horizon and Cauchy horizon of a Kerr black hole as a detailed example.

From the entropy product Equation (22) and entropy sum Equation (23), one can find:

$$
\begin{aligned}
S_{-} d S_{+}+S_{+} d S_{-} & =8 \pi^{2} J d J, \\
d S_{+}+d S_{-} & =8 \pi M d M .
\end{aligned}
$$

This leads to:

$$
\begin{aligned}
& d S_{+}=-\frac{8 \pi^{2} J}{S_{+}-S_{-}} d J+\frac{8 \pi M S_{+}}{S_{+}-S_{-}} d M \\
& d S_{-}=\frac{8 \pi^{2} J}{S_{+}-S_{-}} d J-\frac{8 \pi M S_{-}}{S_{+}-S_{-}} d M .
\end{aligned}
$$

Then, using the entropy minus relation Equation (24), this can be transformed to:

$$
\begin{aligned}
& d M=+T_{+} d S_{+}+\frac{\pi J}{M S_{+}} d J \\
& d M=-\hat{T}_{-} d S_{-}+\frac{\pi J}{M S_{-}} d J
\end{aligned}
$$

where we have used the relation of the Hawking temperature of the Cauchy horizon [15]:

$$
\hat{T}_{-}=-\left.T_{+}\right|_{r_{+} \leftrightarrow r_{-}}=-T_{-}
$$


and $r_{+} \leftrightarrow r_{-}$is the exchange of two horizons. After inserting the relations Equation (26), we obtain the first law of thermodynamics of the event horizon and the Cauchy horizon of a Kerr black hole from the above equations:

$$
\begin{aligned}
& d M=+T_{+} d S_{+}+\Omega_{+} d J \\
& d M=-\hat{T}_{-} d S_{-}+\Omega_{-} d J .
\end{aligned}
$$

On the other hand, the scaling discussion of mass $M\left(S_{+}, J\right)$ gives:

$$
M=2\left(+T_{+} S_{+}+\Omega_{+} J\right) .
$$

For the Smarr relation of the Cauchy horizon, one can assume that:

$$
M=c \hat{T}_{-} S_{-}+d \Omega_{-} J
$$

Using the Hawking temperature of the Cauchy horizon Equation (42) and the dimensionless relation Equation (28), the Smarr relation of the Cauchy horizon leads to:

$$
\begin{aligned}
2 M & =2\left(+T_{+} S_{+}+\Omega_{+} J\right)+\left(-c T_{-} S_{-}+d \Omega_{-} J\right) \\
& =-(2+c) T_{-} S_{-}+\left(2 \Omega_{+}+d \Omega_{-}\right) J .
\end{aligned}
$$

Inserting the relation Equation (27), we find:

$$
2 M=-(2+c) T_{-} S_{-}+\left((d-2) \Omega_{-} J+2 M\right)
$$

implying $c=-2, d=2$, as the mass behaviors as $M\left(S_{-}, \Omega_{-}\right)$in the Cauchy horizon. Finally, we get the Smarr relation of the event horizon and the Cauchy horizon of a Kerr black hole:

$$
\begin{aligned}
& M=2\left(+T_{+} S_{+}+\Omega_{+} J\right) \\
& M=2\left(-\hat{T}_{-} S_{-}+\Omega_{-} J\right) .
\end{aligned}
$$

The above first law of thermodynamics Equation (43) and Smarr relation Equation (44) of the event horizon and the Cauchy horizon of a Kerr black hole are consistent with those in [21].

This discussion can be easily generalized to a Kerr-Newman black hole by substituting $a^{2}$ with $a^{2}+Q^{2}$ in the whole procedure. One can get the following thermodynamic laws:

$$
\begin{gathered}
d M= \pm \hat{T}_{ \pm} d S_{ \pm}+\Omega_{ \pm} d J+\Phi_{ \pm} d Q \\
M=2\left( \pm \hat{T}_{ \pm} S_{ \pm}+\Omega_{ \pm} J\right)+\Phi_{ \pm} Q .
\end{gathered}
$$

Note here that the Hawking temperature of the Cauchy horizon Equation (42) is used, as well. One may expect to generalized this discussion to black holes with three horizons, as well. For example, one can try to obtain the thermodynamic laws of the Schwarzschild-(A)dS black holes by these relations. However, it is not so easy to work out it, as the first law of thermodynamics and the Smarr relation in (A)dS spacetime are still open questions. 


\section{Conclusions}

In this paper, we study the additional thermodynamic relations of black holes with multi-horizons, in order to further understand the origin of black hole entropy at the microscopic level. We obtain some general entropy and temperature relations of multi-horizons, even the "virtual" horizon. We also present how we get these relations in detail. These relations are related to the product, division and sum of the entropy and temperature of multi-horizons. We consider the static and rotating black holes in three and four dimensions. Some general thermodynamic relations are constructed and found to hold for both AdS and dS black holes. Moreover, a new dimensionless and charge-independence relation $\left(\sum_{i=1}^{D} T_{i} S_{i}\right) \times\left(\sum_{i=1}^{D} \frac{1}{T_{i} S_{i}}\right)$ is presented. It does not depend on the mass, electric charge, angular momentum and cosmological constant, because it is a constant for both static and rotating black holes. As it is the generalizing of the $T_{+} S_{+}=T_{-} S_{-}$relations, this relation is expected to be helpful for constructing the black hole/CFT (BH/CFT) correspondence for more than two horizons. Besides, this dimensionless relation is invariant in an inverse transformation $T S \rightarrow \frac{1}{T S}$. This is a symmetry, even if the underlying physical picture is not clear.

On the other hand, the improvement of the topic of the thermodynamic relations is little, while some attempts are shown in this work. Based on the thermodynamic relations presented in this paper, we obtain some thermodynamic bounds of the thermodynamic quantities, including the entropy and temperature. Especially for the upper area bound of the event horizon of black holes, one can find that it is actually the exact Penrose inequality of a black hole, which is the first geometrical inequality of black holes. We also get the Penrose-like inequality of the other horizons. The thermodynamic bounds can be seen as an application of the thermodynamic relations, while how they link to the thermodynamics of black holes seems to be still unclear. However, based on these thermodynamic relations, as well, we present the thermodynamics law and Smarr relation of the event horizon and the Cauchy horizon of a Kerr black hole as a detailed example. This discussion is generalized to Kerr-Newman black holes, as well. These applications of thermodynamic relations also indicate that the thermodynamics of the inner horizon is linked closely with that of the event horizon. This is consistent with the black hole/CFT correspondence [15].

It is also interesting to generalize this discussion about the close relationships between the thermodynamics of multi-horizons to that of three and higher dimensional black holes and other black holes with multi-horizons, including the thermodynamic bound and the thermodynamics laws; especially for black holes in (A)dS spacetime (e.g., Schwarzschild-(A)dS black holes), for which the first law of thermodynamics and the Smarr relation are still open questions. An interesting idea is treating the cosmological constant as a dynamic viable (see, e.g., [53-60]). This may be possibly checked in this way. These are all left as future tasks.

\section{Acknowledgments}

We benefited greatly from discussions with Mirjam Cvetic during the Kavli Institute for Theoretical Physics China (KITPC) Project around June, 2014. Wei Xu would like to thank Geoffrey Compère, Hong Lü and Jian-wei Mei for useful conversations. This work is partially supported by the Natural Science Foundation of China (NSFC) under Grant No. 11075078 and by the Project of Knowledge Innovation 
Program of the Chinese Academy of Sciences. Wei Xu was supported by the Research Innovation Fund of Huazhong University of Science and Technology (2014TS125).

\section{Author Contributions}

The authors have all contributed to the analytical calculations and writing of the paper.

\section{Conflicts of Interest}

The authors declare no conflict of interest.

\section{References}

1. Strominger, A.; Vafa, C. Microscopic origin of the Bekenstein-Hawking entropy. Phys. Lett. B 1996, 379, 99-104.

2. Guica, M.; Hartman, T.; Song, W.; Strominger, A. The Kerr/CFT Correspondence. Phys. Rev. D 2009, 80, 124008.

3. Corda, C. Effective temperature, Hawking radiation and quasinormal modes. Int. J. Mod. Phys. D 2012, 21, 1242023.

4. Corda, C. Black hole quantum spectrum. Eur. Phys. J. C 2013, 73, 2665.

5. Corda, C. Time-Dependent Schrodinger Equation for Black Hole Evaporation: No Information Loss. Ann. Phys. 2015, 353, 71.

6. Corda, C.; Hendi, S.H.; Katebi, R.; Schmidt, N.O. Effective state, Hawking radiation and quasi-normal modes for Kerr black holes. J. High Energ. Phys. 2013, 1306, 008.

7. Cvetic, M.; Gibbons, G.W.; Pope, C.N. Universal Area Product Formulae for Rotating and Charged Black Holes in Four and Higher Dimensions. Phys. Rev. Lett. 2011, 106, 121301.

8. Toldo, C.; Vandoren, S. Static nonextremal AdS4 black hole solutions. J. High Energ. Phys. 2012, $1209,048$.

9. Cvetic, M.; Lu, H.; Pope, C.N. Entropy-Product Rules for Charged Rotating Black Holes. Phys. Rev. D 2013, 88, 044046.

10. Lu, H.; Pang, Y.; Pope, C.N. AdS Dyonic Black Hole and its Thermodynamics. J. High Energ. Phys. 2013, 1311, 033.

11. Chow, D.D.K.; Compère, G. Seed for general rotating non-extremal black holes of $N=8$ supergravity. Class. Quant. Grav. 2014, 31, 022001.

12. Detournay, S. Inner Mechanics of 3d Black Holes. Phys. Rev. Lett. 2012, 109, 031101.

13. Castro, A; Rodriguez, M.J. Universal properties and the first law of black hole inner mechanics. Phys. Rev. D 2012, 86, 024008.

14. Visser, M. Quantization of area for event and Cauchy horizons of the Kerr-Newman black hole. $J$. High Energ. Phys. 2012, 1206, 023.

15. Chen, B.; Liu, S.-X.; Zhang, J.-J. Thermodynamics of Black Hole Horizons and Kerr/CFT Correspondence. J. High Energ. Phys. 2012, 1211, 017.

16. Castro, A.; Lapan, J.M.; Maloney, A.; Rodriguez, M.J. Black Hole Monodromy and Conformal Field Theory. Phys. Rev. D 2013, 88, 044003. 
17. Visser, M. Area products for black hole horizons. Phys. Rev. D 2013, 88, 044014.

18. Abdolrahimi, S.; Shoom, A.A. Distorted Five-dimensional Electrically Charged Black Holes. Phys. Rev. D 2014, 89, 024040.

19. Pradhan, P. Area Products and Mass Formula for Kerr Newman Taub Nut Spacetime. 2013, arXiv:1310.7921.

20. Pradhan, P. Black Hole Interior Mass Formula. Eur. Phys. J. C 2014, 74, 2887.

21. Castro, A.; Dehmami, N.; Giribet, G.; Kastor, D. On the Universality of Inner Black Hole Mechanics and Higher Curvature Gravity. J. High Energ. Phys. 2013, 1307, 164.

22. Faraoni, V.; Moreno, A.F.Z. Are quantization rules for horizon areas universal? Phys. Rev. D 2013, $88,044011$.

23. Lu, H. Charged dilatonic ads black holes and magnetic $\operatorname{AdS}_{D-2} \times R^{2}$ vacua. J. High Energ. Phys. 2013, 1309, 112.

24. Anacleto, M.A.; Brito, F.A.; Passos, E. Acoustic Black Holes and Universal Aspects of Area Products. 2013, arXiv:1309.1486.

25. Xu, W.; Wang, J.; Meng, X.-H. A Note on Entropy Relations of Black Hole Horizons. Int. J. Mod. Phys. A 2014, 29, 1450088.

26. Wang, J.; Xu, W.; Meng, X.-H. The Entropy Relations of Black Holes with Multihorizons in Higher Dimensions. Phys. Rev. D 2014, 89, 044034.

27. Wang, J.; Xu, W.; Meng, X.-H. The "universal property" of horizon entropy sum of black holes in four dimensional asymptotical (anti-)de-Sitter spacetime background. J. High Energ. Phys. 2013, 1401, 031.

28. Xu, W.; Wang, J.; Meng, X.-H. The Entropy Sum of (A)dS Black Holes in Four and Higher Dimensions. Int. J. Mod. Phys. A 2014, 29, 1450172.

29. Du, Y.-Q.; Tian, Y. The Universal Property of the Entropy Sum of Black Holes in All Dimensions. Phys. Lett. B 2014, 739, 250-255.

30. Cvetic, M.; Larsen, F. General rotating black holes in string theory: Grey body factors and event horizons. Phys. Rev. D 1997, 56, 4994.

31. Cvetic, M.; Larsen, F. Grey body factors for rotating black holes in four-dimensions. Nucl. Phys. B 1997, 506, 107-120.

32. Cvetic, M.; Larsen, F. Greybody Factors and Charges in Kerr/CFT. J. High Energ. Phys. 2009, 09, 088.

33. Ansorg, M.; Hennig, J.; Cederbaum, C. Universal properties of distorted Kerr-Newman black holes. Gen. Rel. Grav 2011, 43, 1205-1210.

34. Hennig, J.; Ansorg, M. The Inner Cauchy horizon of axisymmetric and stationary black holes with surrounding matter in Einstein-Maxwell theory: Study in terms of soliton methods. Ann. Henri Poincare 2009, 10, 1075-1095.

35. Ansorg, M.; Hennig, J. The Inner Cauchy horizon of axisymmetric and stationary black holes with surrounding matter in Einstein-Maxwell theory. Phys. Rev. Lett. 2009, 102, 221102.

36. Hennig, J.; Cederbaum, C.; Ansorg, M. A Universal inequality for axisymmetric and stationary black holes with surrounding matter in the Einstein-Maxwell theory. Commun. Math. Phys. 2010, 293, 449-467. 
37. Ansorg, M.; Hennig, J. The Inner Cauchy horizon of axisymmetric and stationary black holes with surrounding matter. Class. Quant. Grav. 2008, 25, 222001.

38. Ansorg, M.; Pfister, H. A Universal constraint between charge and rotation rate for degenerate black holes surrounded by matter. Class. Quant. Grav. 2008, 25, 035009.

39. Jaramillo, J.L.; Vasset, N.; Ansorg, M. A Numerical study of Penrose-like inequalities in a family of axially symmetric initial data. 2007, arXiv:0712.1741.

40. Booth, I.; Fairhurst, S. Extremality conditions for isolated and dynamical horizons. Phys. Rev. D 2008, 77, 084005.

41. Hennig, J.; Neugebauer, G. Non-existence of stationary two-black-hole configurations. 2010, arXiv: 1002.1818 .

42. Chen, B.; Zhang, J.-J. Holographic Descriptions of Black Rings. J. High Energ. Phys. 2012, 1211, 022.

43. Chen, B.; Zhang, J.-J. RN/CFT Correspondence From Thermodynamics. J. High Energ. Phys. 2013, 1301, 155.

44. Chen, B.; Zhang, J.-J. Electromagnetic Duality in Dyonic RN/CFT Correspondence. Phys. Rev. D 2013, 87, 081505.

45. Chen, B.; Xue, Z.; Zhang, J.-J. Note on Thermodynamic Method of Black Hole/CFT Correspondence. J. High Energ. Phys. 2013, 1303, 102.

46. Chen, B.; Zhang, J.-J.; Zhang, J.-D.; Zhong, D.-L. Aspects of Warped $\mathrm{AdS}_{3} / \mathrm{CFT}_{2}$ Correspondence. J. High Energ. Phys. 2013, 1304, 055.

47. Chen, B.; Zhang, J.-J. Thermodynamics in Black-hole/CFT Correspondence. Int. J. Mod. Phys. D 2013, 22, 1342012.

48. Okamoto, I.; Kaburaki, O. The 'inner-horizon thermodynamics' of Kerr black holes. Mon. Not. R. Astron. Soc. 1992, 255, 539-544.

49. Mars, M. Present status of the Penrose inequality. Class. Quant. Grav. 2009, 26, 193001.

50. Bekenstein, J.D. Black holes and the second law. Lett. Nuovo Cim 1972, 4, 737-740.

51. Hawking, S.W. Particle Creation by Black Holes. Commun. Math. Phys. 1975, 43, 199-120; Erratum in 1976, 46, 206.

52. Banados, M.; Teitelboim, C.; Zanelli, J. The Black hole in three-dimensional space-time. Phys. Rev. Lett. 1992, 69, 1849.

53. Kastor, D.; Ray, S.; Traschen, J. Enthalpy and the Mechanics of AdS Black Holes. Class. Quant. Grav. 2009, 26, 195011.

54. Dolan, B.P. Pressure and volume in the first law of black hole thermodynamics. Class. Quant. Grav. 2011, 28, 235017.

55. Cvetic, M.; Gibbons, G.W.; Kubiznak, D.; Pope, C.N. Black Hole Enthalpy and an Entropy Inequality for the Thermodynamic Volume. Phys. Rev. D 2011, 84, 024037.

56. Dolan, B.P.; Kastor, D.; Kubiznak, D.; Mann, R.B.; Traschen, J. Thermodynamic Volumes and Isoperimetric Inequalities for de Sitter Black Holes. Phys. Rev. D 2013, 87, 104017.

57. $\mathrm{Xu}, \mathrm{W}$.; Xu, H.; Zhao, L. Gauss-Bonnet coupling constant as a free thermodynamical variable and the associated criticality. Eur. Phys. J. C 2014, 74, 2970.

58. Xu, H.; Xu, W.; Zhao, L. Extended phase space thermodynamics for third order Lovelock black holes in diverse dimensions. Eur. Phys. J. C 2014, 74, 3074. 
59. Xu, W.; Zhao, L. Critical phenomena of static charged AdS black holes in conformal gravity. Phys. Lett. B 2014, 736, 214-220.

60. Altamirano, N.; Kubiznak, D.; Mann, R.B.; Sherkatghanad, Z. Thermodynamics of rotating black holes and black rings: Phase transitions and thermodynamic volume. Galaxies 2014, 2, 89-159.

(c) 2015 by the authors; licensee MDPI, Basel, Switzerland. This article is an open access article distributed under the terms and conditions of the Creative Commons Attribution license (http://creativecommons.org/licenses/by/4.0/). 